An official journal of the / Un journal officiel de la "Société Sénégalaise de Cancérologie" (SOSECAN)

Journal homepage: www.africanjournalofoncology.com

DOI: https://www.doi.org/I0.54266/ajo.1.2.60.JFIC48I3

\title{
Undifferentiated carcinoma of the nasopharynx associated with an early paraneoplastic dermatomyositis TINOMO: About a one case
}

\section{Carcinome indifférencié du nasopharynx associé à une dermatomyosite paranéoplasique à un stade précoce TINOMO : A propos d'un cas et revue de la littérature}

S. Panandtigri'*, M. Charkaoui', NPX. Sia', N. Benchakroun', C. Tarek', M. Bourhafour', Z. Bouchbika', H. Jouhadi', N. Tawfiq', S. Saharaoui', A. Benider'.

I Centre Mohamed VI pour le traitement des cancers du Centre Hospitalier Universitaire lbn Rochd de Casablanca, Maroc.

\begin{abstract}
Dermatomyositis (DM) is a rare autoimmune disease characterized by non-infectious inflammatory damage to the skin and the predominant muscles in the belts. It is believed to be associated with about one case per I,000 nasopharyngeal carcinoma (NPC). This association has been described for the locally advanced stage, stage II and stage III of the NPC. Little described in the early stages (stage I), its symptomatology is often indicative of cancer whose diagnosis is based on five criteria: muscle weakness of the proximal limbs, elevation of serum muscle enzymes, histological muscle abnormalities in muscle biopsy, electromyographic abnormality and clinical inflammatory dermatological manifestation. Treatment is based on corticosteroid therapy and etiological treatment of cancer. The prognosis of NPC in patients with paraneoplastic DM is comparable to noninfected patients. We report this case of DM revealing an early stage NPC and we take stock of the state of knowledge about paraneoplastic DM associated with rarely described NPC. Indeed, in Morocco the last description of a case dates back to 2016.
\end{abstract} KEYWORDS: Dermatomyositis; Paraneoplastic; Nasopharynx; Corticosteroid therapy; Radiotherapy.

RESUME : La dermatomyosite (DM) est une maladie auto-immune rare, caractérisée par une atteinte inflammatoire non infectieuse de la peau et des muscles prédominants aux ceintures. Elle serait associée à environ un cas sur 1.000 au carcinome nasopharyngé (NPC). Cette association a été décrite pour les stades localement avancés, stade II et stade III du NPC. Peu décrite à des stades précoces (stade I), sa symptomatologie est souvent révélatrice du cancer dont le diagnostic repose sur cinq critères : la faiblesse musculaire des membres proximaux, l'élévation des enzymes musculaires sériques, les anomalies musculaires histologiques à la biopsie musculaire, l'anomalie électromyographique et la manifestation dermatologique inflammatoire clinique. Le traitement repose sur la corticothérapie et le traitement étiologique du cancer. Le pronostic des NPC chez les patients atteints de DM paranéoplasique est comparable aux patients non atteints. Nous rapportons ce cas de DM révélant un NPC à un stade précoce et nous faisons le point sur l'état des connaissances sur la DM paranéoplasique associée à un NPC rarement décrite. En effet, au Maroc la dernière description d'un cas remonte en 2016.

MOTS-CLES : Dermatomyosite ; Paranéoplasie ; Nasopharynx ; Corticothérapie ; Radiothérapie.

\section{INTRODUCTION}

La dermatomyosite (DM) est une maladie auto-immune rare, caractérisée par une atteinte inflammatoire non infectieuse de la peau et des muscles prédominants aux ceintures [ $\mathrm{I}$ ]. Elle est associée à une tumeur maligne dans 18 à $32 \%$ des cas, apparaissant avant, simultanément ou après le diagnostic du cancer [2]. Ces cancers sont notamment des cancers ovariens, bronchiques, du sein, de la région de la tête et du cou ou plus rarement des lymphomes.

En effet, l'incidence de DM associée à un carcinome pharyngé (NPC) est de l'ordre d'un pour 1.000 cas de NPC [3]. Cette association a été décrite pour les stades localement avancés, stade II [3] et stade III [4] du NPC. L'objectif de la présente étude est de rapporter un cas de DM paranéoplasique révélant un carcinome indifférencié du nasopharynx (UCNT) à un stade précoce (stade I). Nous décrirons par ailleurs les aspects diagnostics, thérapeutiques et pronostiques de cette entité, bien que rare, qui pourrait aider les cliniciens à un diagnostic précoce de ces cancers.

\section{OBSERVATION}

II s'agit d'un patient de 65 ans, sans antécédents particuliers référé au Centre Mohamed VI pour le traitement des cancers du Centre Hospitalier Universitaire ( $\mathrm{CHU}$ ) Ibn Rochd de Casablanca, pour prise en charge d'un cancer du nasopharynx. Le début de sa symptomatologie remonte à trois mois par l'apparition d'une dysphagie au solide intermittente, puis devenue totale, motivant la mise en place d'une sonde nasogastrique d'alimentation, associée à une éruption cutanée au visage et aux mains avec une fatigue intense et un amaigrissement important non chiffré. A l'examen clinique, nous retrouvions un patient très altéré avec un Performans Status (PS) à trois, un érythème au niveau du visage, du cou et des extrémités avec un score CDASI (Cutanéous Dermatomyositus Disease Area and Severity Index) à 15 (Figures I, 2 et 3). L'examen révélait aussi un déficit musculaire au niveau des muscles axiaux coté à huit selon le MMT (Manual Muscle Testing) ; les muscles proximaux à 32 et distaux à huit avec un score global MMT à 48 sur 260 [I]. 


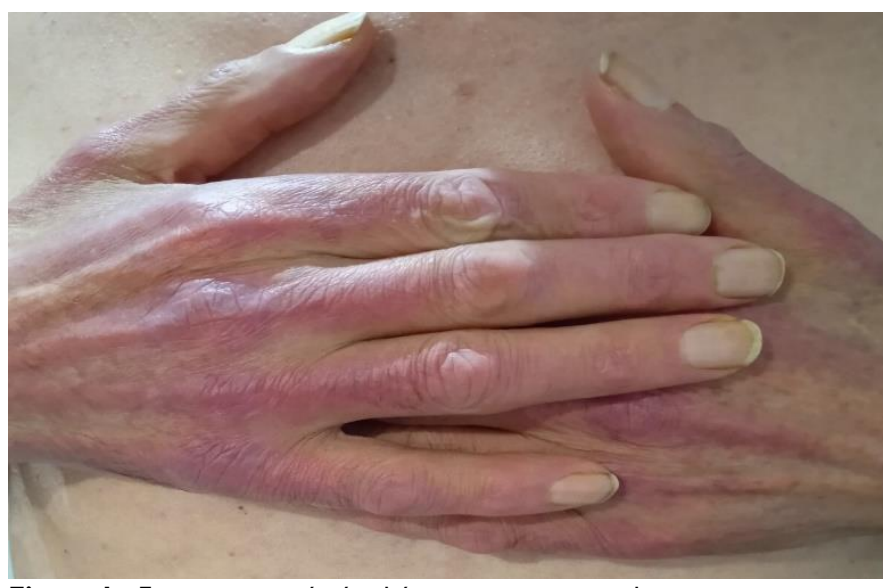

Figure I : Eruption cutanée érythémateuse au niveau des mains.

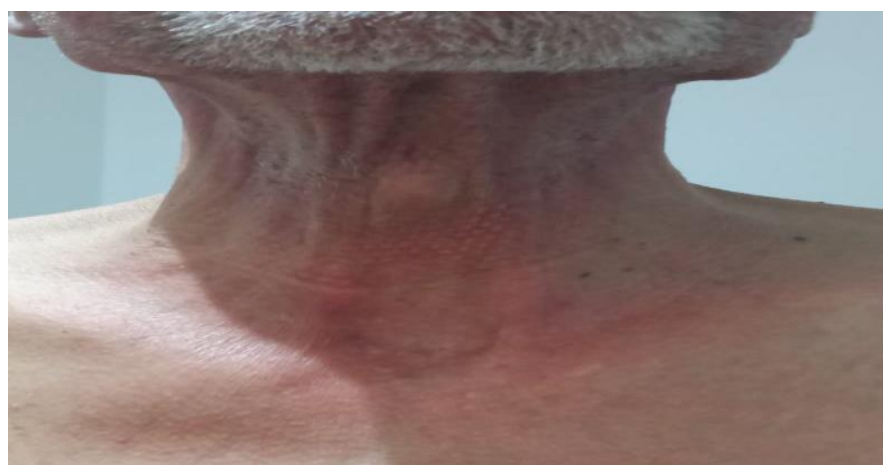

Figure 2 : Eruption cutanée érythémateuse au niveau de la face antérieure du cou.

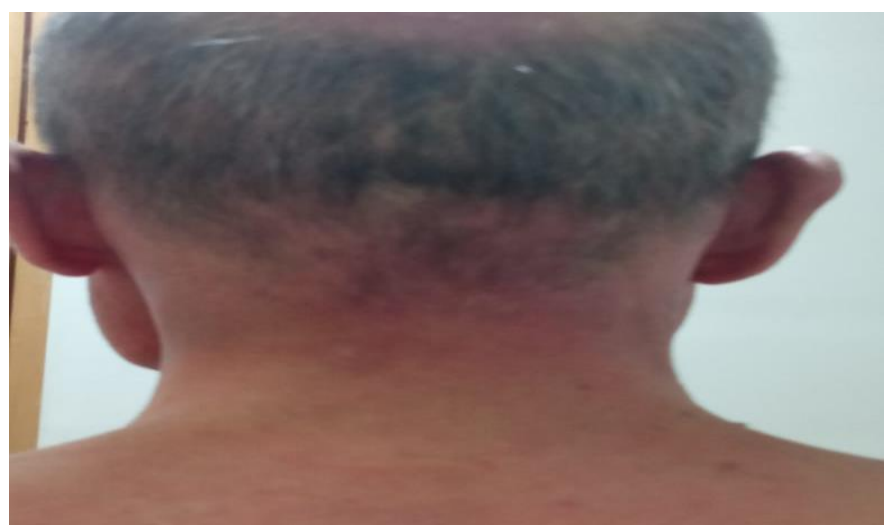

Figure 3 : Eruption cutanée érythémateuse au niveau de la face postérieure du cou.

Le bilan para-clinique pour le diagnostic du cancer était fait de panendoscopie montrant un processus tumoral au niveau de la paroi postérieure du nasopharynx et la biopsie retrouvait un UCNT, confirmé à l'immunohistochimie par expression de P63. Un bilan radiologique fait d'imagerie par résonnance magnétique (IRM) du nasopharynx et d'une tomographie par émission de positon (TEP) a permis de poser le diagnostic de UCNT du nasopharynx stade I (TINOM0). Devant les signes cliniques évocateurs de DM, un bilan biologique demandé avait montré l'élévation du lactate déshydrogénase (LDH) : 30 I $\mathrm{UI} / \mathrm{L}$; avec créatine phosphokinnase (CPK): $148 \mathrm{UI} / \mathrm{L}$ (normale) et les anticorps anti-nucléaires positifs à I/640 de types homogènes.

Le diagnostic final de DM paranéoplasique avec un IMACS (évaluation globale de l'activité de la maladie par le médecin) évalué sur une échelle de Likert à quatre correspondant à une maladie active très sévère, associé à un UCNT du nasopharynx stade I chez un patient altéré a été posé avec nécessité de débuter un traitement de la DM du fait de son activité extrême [I]. Le patient a bénéficié d'une corticothérapie à la dose de I $\mathrm{mg} / \mathrm{kg} / \mathrm{j}$ en injectable pendant trois jours, puis relais par voie orale pendant quatre mois et dégression lente de 2,5 $\mathrm{mg} / \mathrm{semaine}$. L'évolution fut marquée par une amélioration spectaculaire sous corticothérapie: PS à deux, ablation de la sonde nasogastrique d'alimentation et une nette régression des lésions cutanées avec un score CDASI à II ; une diminution du déficit moteur avec un MMT260 à 208 et baisse de moitié des valeurs des enzymes musculaires LDH : $160 \mathrm{UI} / \mathrm{L}$; CPK : $40 \mathrm{UI} / \mathrm{L}$. Ensuite, le traitement de son cancer a été instauré à savoir une radiothérapie exclusive à raison de $70 \mathrm{~Gy}$ en dose totale sur la tumeur en fractionnement de 2 Grays (Gy)/fraction (fr) en 35 séances de cinq séances/semaine. A la fin du traitement étiologique, nous retrouvions un patient autonome (PS à un I) avec une disparition des lésions cutanées (Figure 4) et récupération totale de la force musculaire.

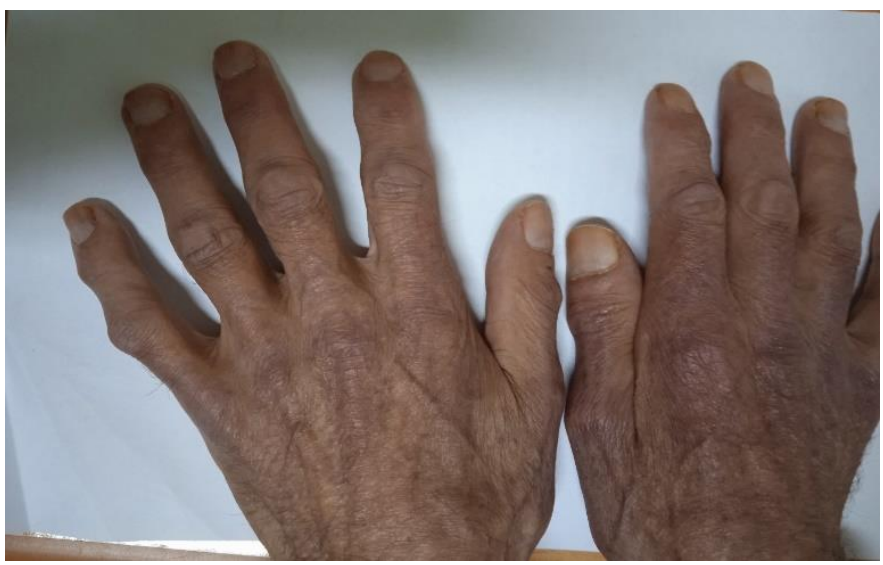

Figure 4 : Disparition des lésions cutanées.

\section{DISCUSSION}

Les carcinomes nasopharyngés (NPC) représentent moins de $1 \%$ de toutes les tumeurs malignes et affectent environ une personne sur 100.000 en Amérique du Nord et en Europe Occidentale, mais est plus fréquente en Asie et en Afrique du Nord. Ils affectent cinq à neuf personnes pour 100.000 habitants [5]. Le sous-type pathologique indifférencié est le plus fréquent avec une association fréquente avec des syndromes paranéoplasiques comme la fièvre, les réactions leucémoides d'ostéosthropathie [6]. L'association DM et cancer est fréquente allant de 18 à $32 \%$ [2]. Cependant son association avec le NPC est peu décrite. Le plus souvent, elle est associée au type indifférencié, un peu moins fréquemment au type peu différencié et jamais au cancer bien différencié [7]. Elle peut être plus grave que les conséquences de la tumeur primitive elle-même et peut précéder, suivre ou être concomitant au diagnostic du cancer. C'est le constat dans notre cas où la symptomatologie est dominée par la DM. La pathogénèse de la DM est encore mal connue et plusieurs mécanismes ont été suggérés. Deux théories ont été développées: la théorie hormonale et la théorie immunologique. Dans la première hypothèse, c'est la tumeur qui secrète des polypeptides hormonaux actifs sur le plan biologique mais inappropriés sur le plan homéostatique. 
Ces polypeptides seraient responsables des différents syndromes cliniques de types endocriniens [7]. Dans la théorie immuno-allergique, le syndrome paranéoplasique serait le résultat de réactions croisées des anticorps produits contre les antigènes tumoraux, avec les tissus normaux ayant une similitude de structures [7]. Le diagnostic de DM est basé sur cinq critères selon Bohan et Peter [8] : la faiblesse musculaire progressive et symétrique des ceintures et des muscles fléchisseurs du cou; des signes dermatologiques (éruptions cutanées à l'héliotrope avec œdèmes périorbitaires, papules de Grotton (dermatite squameuse sur les articulations des doigts), dermatite sur les coudes, les genoux et les pieds); une biopsie musculaire en faveur de myosites; une élévation des enzymes musculaires sériques témoignant de la nécrose musculaire (en particulier CPK, aldolase, LDH), ils sont augmentés chez 70 à $90 \%$ des patients, cependant un taux normal ne doit pas faire écarter le diagnostic [I] et le profil électromyographique en faveur d'une atteinte musculaire. La présence de trois ou quatre de ces critères, en plus de l'éruption cutanée permet de faire le diagnostic de DM et la présence de deux critères associés à l'éruption cutanée est très évocatrice de DM. Ainsi, devant la faiblesse musculaire progressive, l'élévation de la LDH associée à l'éruption cutanée typique, nous avons posé le diagnostic de DM paranéoplasique. La DM peut être idiopathique, en particulier chez les enfants. Mais elle est généralement liée à une tumeur maligne. Dans l'étude épidémiologique de Hill et al [2], 32\% des DM étaient associées à des cancers de l'ovaire, du poumon, du pancréas, du sein, du tractus gastro-intestinal ou à un lymphome non hodgkinien. Egalement, Chan et al [9] ont publié une série de cas de DM suivie à Singapour, dont $41 \%$ étaient des carcinomes du nasopharynx. Hu et al [10], dans une étude statistique en Chine trouvaient une association de la DM au cancer avec une incidence de $20.3 \%$ dont $78.5 \%$ concernait le NPC. Le traitement de la DM chez un patient atteint de cancer, implique le traitement de la DM et le traitement de la tumeur. II est identique chez les patients avec ou sans cancer associé. Le but de la thérapeutique dans la DM, est d'augmenter la force musculaire et d'améliorer les manifestations extra-musculaires. Le traitement en première intention est basé sur la corticothérapie à forte dose associée aux mesures hygiénodiététiques usuelles de la corticothérapie [II]. En effet, avant l'utilisation des corticoïdes, le pronostic des DM était médiocre avec une mortalité de 50 à $60 \%$ [12].

Les corticoïdes sont prescrits à la dose de $\mathrm{I} \mathrm{mg} / \mathrm{kg} / \mathrm{j}$ pendant quatre à huit semaines en moyenne, jusqu'à régression des signes cliniques et la nette diminution ou la normalisation des enzymes musculaires, puis une dégression progressive peut être entamée jusqu'à l'obtention de la dose minimale efficace qui sera maintenue pendant six à neuf mois sans rechute [13]. Pour les formes sévères de DM, l'utilisation de bolus intraveineux de méthyl-prednisolone à la dose de I mmg/kg/j pendant trois jours, puis relais par voie orale est recommandée [12]. Ce fut le cas pour notre patient admis dans un état altéré qui a reçu un bolus en injectable, puis relais par voie orale. Cependant en cas de DM réfractaire ou corticodépendance, d'autres options thérapeutiques sont utilisées telles que le méthotrexate, l'azthioprine, les immunoglobulines intraveineuses et rituximab [14]. En plus de la corticothérapie, il faut associer le traitement du NPC. S'agissant du stade I, le traitement est la radiothérapie exclusive de 70 Gy en fractionnement de $2 \mathrm{~Gy} / \mathrm{fr}$ en 35 séances, cinq séances/semaine comme dans notre cas. Le pronostic du NPC avec DM est le même que celui d'un NPC en général. En effet, Hu et al, dans une étude cas-témoins, montraient un taux de survie de cinq et dix ans et un taux de métastase à distance, respectivement de 50,$4 ; 34,5$ et $40,5 \%$ pour les patients NPC atteints de DM et de 57,$8 ; 55,2$ et $56,5 \%$ pour les témoins non-atteints de DM. Les résultats montraient que la radiothérapie à la prednisone est un traitement non-seulement assez efficace, mais aussi n'entrainerait pas non plus une augmentation significative du taux de métastases à distance [10]. En plus, de nombreux auteurs ont rapporté qu'une rémission complète après une radiothérapie sur la tumeur entrainait la disparition ou l'amélioration des symptômes et des signes physiques du DM. Ainsi, la rechute du DM était corrélée à une récidive locorégionale ou à la détection de métastases, constituant alors un élément de surveillance après traitement de NPC.

\section{CONCLUSION}

Les NPC avec DM paranéoplasique sont une entité rare. Le diagnostic de DM est basé sur cinq critères cliniques et paracliniques. II est parfois révélateur de certains NPC, constituant alors un élément aussi bien de diagnostic précoce que de surveillance. Ainsi, devant une DM survenant chez des malades originaires des zones endémiques, le NPC doit être recherché en priorité. En plus, l'association du DM avec le NPC ne semble pas influencer le pronostic du cancer, d'où l'intérêt d'une prise en charge rapide de la DM pour améliorer la qualité de vie du patient et lui permettre de supporter les traitements spécifiques du cancer.

\section{CONFLITS D'INTERET}

Les auteurs n'ont déclaré aucun conflit d'intérêts.

\section{REFERENCES}

I. Brigitte Bader-Meunier et Olivier Benveniste. Protocole National de Diagnostic et de Soins (PNDS) Dermatomyosite de l'enfant et de l'adulte. Juillet 2016. 10.

www.has-sante.fr.

2. Hill, C. L., Y. Zhang, B. Sigurgeirsson, E. Pukkala, L. Mellemkjaer, A. Airio, S. R. Evans, et D. T. Felson. « Frequency of Specific Cancer Types in Dermatomyositis and Polymyositis : A PopulationBased Study ». Lancet (London, England) 357, no 9250 (I3 janvier 200I) : 96 I00. https://doi.org/10.1016/S0140-6736(00)03540-6.

3. Ziani, Fatima Zahra, Sami Aziz Brahmi, Rajae Najib, Rajae Kanab, Samia Arifi, Fatima Zahra Mernissi, et Nawfal Mellas. « [Paraneoplastic dermatomyositis revealing an undifferentiated nasopharyngeal carcinoma : about a case] ». The Pan African Medical Journal 24 (2016) : 29.

https://doi.org// 0.1 I604/pamj.2016.24.29.4822.

4. Chakroun, A., J. Guigay, A. Lusinchi, P. Marandas, F. Janot, et D.M. Hartl. " Paraneoplastic Dermatomyositis Accompanying Nasopharyngeal Carcinoma : Diagnosis, Treatment and Prognosis ». European Annals of Otorhinolaryngology, Head and Neck Diseases 128, no 3 (juin 20II) : I 2731. https://doi.org/10.1016/j.anorl.2010.10.007.

5. Vasef, Mohammad A., Alfio Ferlito, et Lawrence M. Weiss. « Nasopharyngeal Carcinoma, with Emphasis 
on Its Relationship to Epstein-Barr Virus ». Annals of Otology, Rhinology \& Laryngology 106, no 4 (avril 1997) : 34856.

https://doi.org//0.1 I77/0003489497/06004/6.

6. Toro, C., A. Rinaldo, C.E. Silver, M. Politi, et A. Ferlito. « Paraneoplastic Syndromes in Patients with Nasopharyngeal Cancer ». Auris Nasus Larynx 36, no 5 (octobre 2009) : 51320.

https://doi.org/l0.1016/j.anl.2008.10.006.

7. Maalej, M., A. Ladgham, A. Ennouri, A. Ben Attia, M. Cammoun, et R. Ellouze. " [The paraneoplastic syndrome in nasopharynx cancer. 32 cases] 11 . Presse Medicale (Paris, France : 1983) 14, no 8 (23 février 1985) : 47। 74.

8. Bohan, A., et J. B. Peter. « Polymyositis and Dermatomyositis (First of Two Parts) ॥. The New England Journal of Medicine 292, no 7 ( 3 février 1975) : 34447.

https://doi.org/10.1056/NEJMI 97502 I 32920706.

9. Chan, H. L. " Dermatomyositis and Cancer in Singapore ». International Journal of Dermatology 24, no 7 (septembre 1985) : 44750. https://doi.org/ | 0.1 | | I/j.| 365-4362.1985.tb058|6.x.

10. Wj, Hu, Chen DI, et Min Hq. "Study of 45 cases of nasopharyngeal carcinoma with dermatomyositis ». American journal of clinical oncology. Am J Clin Oncol, février 1996.

https://doi.org/10.1097/0000042 I-199602000-00008.

I I. Frikha, Faten, Mouna Snoussi, Raida Ben Salah, Noura Saidi, Neila Kaddour, et Zouhir Bahloul. « [Dermatomyositis in the elderly : a study of 4 cases in southern Tunisia] ». The Pan African Medical Journal 13 (20I2) : 26.

12. Marie, Isabelle, et Luc Mouthon. " Therapy of Polymyositis and Dermatomyositis ». Autoimmunity Reviews II, no I (I novembre 20 I I) : 6 I3. https://doi.org/10.1016/j.autrev.2011.06.007.

13. Chérin, P. « [Current therapy for polymyositis and dermatomyositis] ॥. La Revue De Medecine Interne 29 Spec No 2 (juin 2008) : 9 I4.

14. Pautas, E., P. Chérin, J. C. Piette, S. Pelletier, B. Wechsler, J. Cabane, et S. Herson. "Features of Polymyositis and Dermatomyositis in the Elderly: A Case-Control Study ». Clinical and Experimental Rheumatology 18, no 2 (avril 2000) : 24I 44. 


\section{DECLARATIONS}

- Approbation éthique et consentement à participer : sans objet.

- Consentement à la publication : sans objet.

- Disponibilité des données et du matériel : sans objet.

- Intérêts concurrents : les auteurs ne déclarent aucun conflit d'intérêts.

- $\quad$ Financement : sans objet.

- Contributions des auteurs : tous les auteurs ont contribué à la rédaction et l'élaboration de cet article. Ils ont également tous lu et approuvé la version finale du manuscript.

- $\quad$ Remerciements : sans objet. 